in the ratio both of new capital and of new production capacity to man-power represented by the new schemes, and estimates that in 1951 the output of the industry should be 50 per cent greater than in 1946. On completion of the expansion schemes, a reduction of imports by $£ 28$ millions is forecast by the manufacturers; but this is regarded as too high, as is the estimate of a $£ 49$ millions increase in exports (including plastics). Nevertheless, on the assumption that the plans of the industry for rehabilitation and expansion are accomplished promptly, and subject to certain reservations about sulphuric acid, it is believed that, so far as can be foreseen, the industry will prove able to meet not only the full needs of the home market, but also to eliminate avoidable chemical imports and provide a large increase in exports. It is unlikely that the expenditure on research is exceeded in any other industry in Great Britain, and the figure compares well with corresponding figures in other countries, such as the United States. The report maintains that there is evidence that the industry has within itself the resources, vigour and foresight to meet the foreseeable future and also to maintain its position in the longer term. In conclusion, it is urged that in this immensely complicated industry or series of indus. tries, which is so often a continuous adventure into the unknown, the national interest will best be served by the utmost freedom of enterprise.

\section{ORIGIN OF THE FLUCTUATIONS IN THE INTENSITY OF RADIO WAVES FROM GALACTIC SOURCES}

\section{Cambridge Observations}

$\mathrm{T}$ HE discovery by Hey, Parsons and Phillips ${ }^{1}$ of a variable component of the radio emission from the constellation of Cygnus suggested that at least part of the galactic radiation was due to discrete sources of small angular diameter. Later observations by Bolton and Stanley ${ }^{2}$ and by Ryle and Smith ${ }^{3}$ showed that the source subtended an angle of less than 8 minutes of arc. Ryle ${ }^{4}$ has summarized the evidence concerning the nature of the sources, and has shown that if the observed fluctuations are due to variations of the emission from the source, and are not the result of some diffraction mechanism in the earth's atmosphere, it is possible to deduce the maximum physical dimensions of the source from the observed period of the fluctuations. It is therefore of importance to determine whether the observed fluctuations are caused by some relatively local diffraction mechanism, or whether they represent genuine variations of the emission from the source.

In a private communication, Dr. J. L. Pawsey has described some experiments by Bolton, in which tbe source in Cygnus was observed simultaneously from sites in Australia and New Zealand. The records of fluctuations obtained in these experiments were markedly different at the two sites, and therefore suggested a comparatively local origin. In these experiments, however, the observations were made when the angle of elevation of the source was small; the radiation was therefore incident on the ionosphere

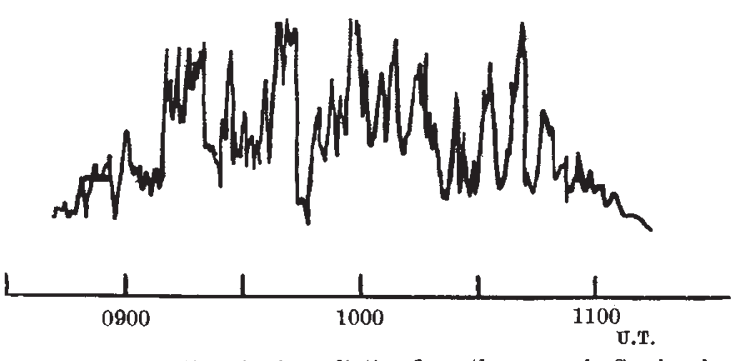

Fig. 1. Fluctuations in the radiation from the source in Cassiopeia on a wave-length of $6.7 \mathrm{~m}$.; October 27,1949

and troposphere at a rather large angle. Since it was possible that the refraction effects in those experiments might cause fluctuations sufficiently large to mask any genuine variations of the incident radiation, it was decided to repeat the experiment using an observing technique which permitted observation of the source with a relatively much smaller angle of incidence $\left(12^{\circ}\right)$, for which the refraction effects should be greatly reduced. It was also possible to make similar observations of the other intense source in the constellation of Cassiopeia, which had been found in previous experiments to be relatively less disturbed.

Two series of experiments have therefore been carried out; the first of these was done in conjunction with Little and Lovell, and involved simultaneous observations on a wave-length of $3.7 \mathrm{~m}$., using receivers at Cambridge and Jodrell Bank, Cheshire (separation $210 \mathrm{~km}$.). A detailed account of these observations is given in the following communication from Little and Lovell; but no detailed correlation between the rapid fluctuations of intensity at the two receivers was found.

The second series of observations was made by the Cambridge workers on a wave-length of $6.7 \mathrm{~m}$., using spacings of up to $170 \mathrm{~km}$. In these observations two types of fluctuations were found.

(1) On many nights, rapid fluctuations similar to those on a wave-length of $3 \cdot 7 \mathrm{~m}$. were observed; no detailed correlation was found when the receivers were separated by more than $20 \mathrm{~km}$. A typical record of this type of fluctuation is shown in Fig. 1. Observations on both $3.7 \mathrm{~m}$. and $6.7 \mathrm{~m}$. have shown that this type of fluctuation involves variations of the intensity both above and below the mean value. This observation suggests strongly that diffraction, such as might be caused in the ionosphere, is responsible for the disturbances, although there are two difficulties in such an explanation. First, it has been found that simultaneous observations at Cambridge on $3.7 \mathrm{~m}$. and $6.7 \mathrm{~m}$. often show considerable cor-

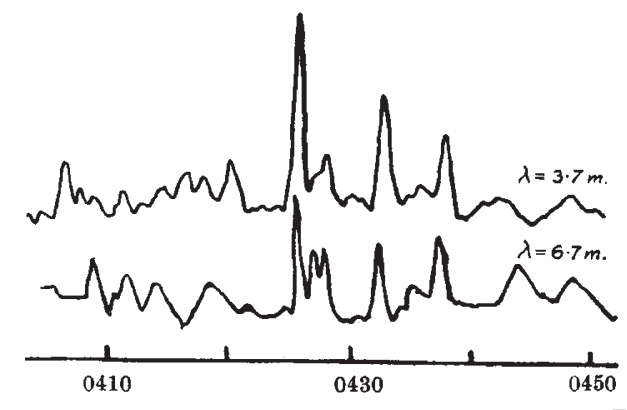

U.T.

Fig. 2. Fluctuations in the radiation from the source in Cassiopeia, showing correlation between wave-lengths of $3.7 \mathrm{~m}$. and $6 \cdot 7 \mathrm{~m}$. 


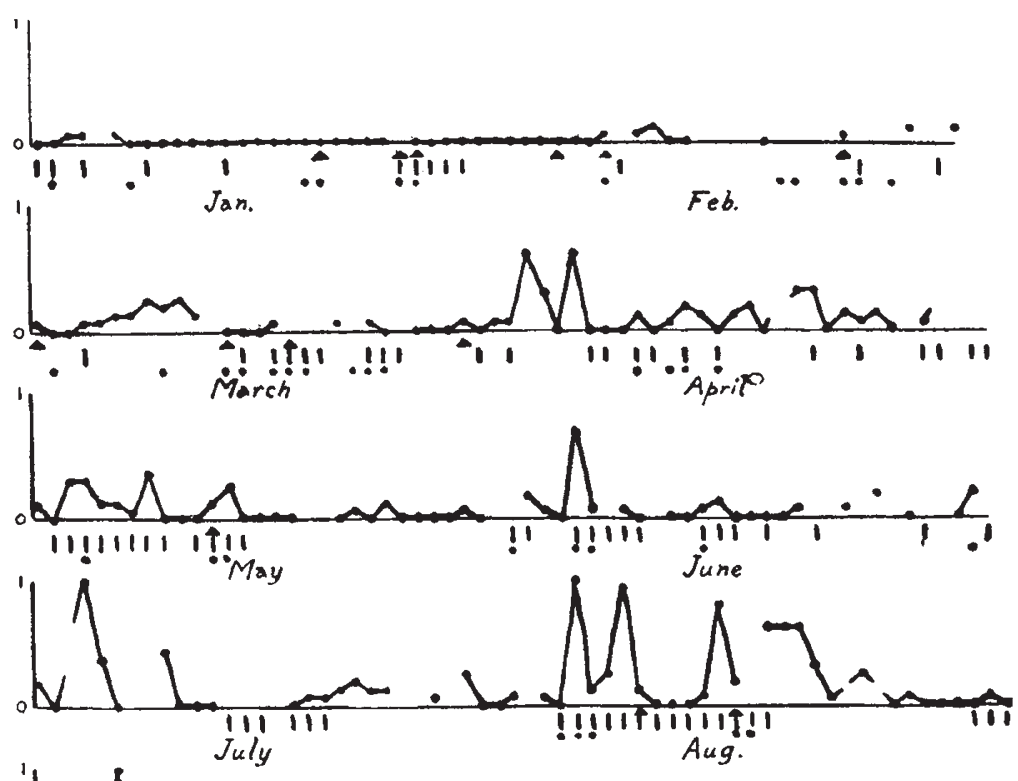

not possible to relate with certainty the direction of origin of the 'bursts' with that of either of the major sources; in view of the large spacing between the receivers, it does not appear likely, however, that the fluctuations could be of terrestrial origin, and the observations suggest that they represent genuine variations of the emission from the galactic sources.

It therefore seems likely that two separate mechanisms are responsible for the observed fluctuations. One appears to be related to varia. tions of the emission from the sources; the brief duration of these 'bursts' suggests that the sources are of stellar dimensions. The other appears to be due to diffraction in a comparatively local region. Further experiments are necessary to confirm both these conclusions.

I should like to express my thanks to Mr. M. Ryle for his advice and help throughout this work, to Messrs. K. E. Machin and B. Elsmore for assistance in making the observations, and to Dr. N. A. de Bruyne, of Aero Research, Ltd., the Chief Superintendent of the Telecommunications Research Establishment, Malvern, and the Officer Commanding, Fig. 3. Intensity of fluctuations expressed as a fraction of the mean intensity (average
for the sources in Cygnus and Cassiopeia). -, magnetic storms

relation (Fig. 2) ; any simple diffraction mechanism would be expected to show markedly different patterns on two such widely separated wave-lengths. Secondly, the results obtained in a series of observations on $3.7 \mathrm{~m}$. over a period of ten months have shown. little correlation with periods when the ionosphere was known to be disturbed. The amplitude of the fluctuations is shown in Fig. 3, together with the occurrence of ionospheric and magnetic storms and auroras.

(2) Occasionally a different type of fluctuation was observed on a wave-length of $6.7 \mathrm{~m}$., which had the appearance of individual 'bursts' of large amplitude and of duration 10-20 sec. Fluctuations of this type were found to show good correlation at even widely spaced receiving sites. Fig. 4 shows simultaneous records obtained with a spacing of $160 \mathrm{~km}$. This type of fluctuation was comparatively rare during the period of the observations, and it was therefore

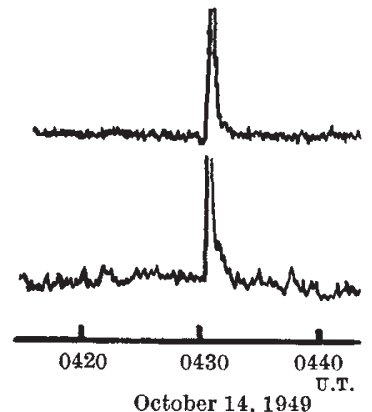

October 14,1949

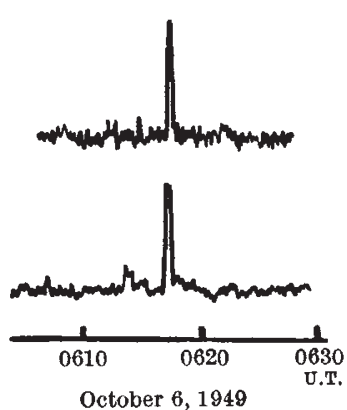

October 6, 1949
Fig. 4. Simultaneous records obtained on a wave-Jength of $6.7 \mathrm{~m}$., with receivers separated by a distance of $160 \mathrm{~km}$. Top traces, Cambridge ; lower traces. Defford (Worcester)
R.A.F., Defford, for facilities in carrying out the spaced receiver observations. The work is part of a programme of research at the Cavendish Laboratory supported by the Department of Scientific and Industrial Research.

Cavendish Laboratory,

F. G. SMTtr Cambridge.

Dec. 9.

${ }^{1}$ Hey, J. S., Parsons, S. J., and Phillips, J. W., Nature, 158, 234 (1946). - Bolton, J. G., and Stanley, G. J., Nature, 161, 312 (1948).

Ryle, M., and Smith, F. G., Nature, 162, 462 (1948).

‘ Ryle, M., Proc. Phys. Soc., A, 62, 491 (1949).

\section{Jodrell Bank Observations}

THE existence of intense sources of radio-frequency radiation in the constellations of Cygnus and Cassiopeia has been established by the measurements of Bolton and Stanley', and Ryle and Smith². The existence of the source in Cygnus had been inferred previously by Hey, Parsons and Phillips ${ }^{2}$ on account of the short-period fluctuations in intensity often shown by the radiation from the locality. Afterwards the source in Cassiopeia was also found to fluctuate ${ }^{2}$, and it has been generally assumed that this variability is inherent in"the source of radiation. Reasons for suspecting this assumption have been given by Smith in the accompanying communication.

In an attempt to settle this question unambiguously, a series of measurements of the radiation from the sources in Cygnus and Cassiopeia have been made in co-operation with Smith between May 1, 1949, and October 31, 1949. In this work the radiation from the 